\title{
Errata
}

\section{A FEW PROPERTIES OF A CERTAIN CLASS OF DEGENERATE} SPACE-TIMES, Jerzy K. Kowalczyński and Jerzy F. Plebański, International Journal of Theoretical Physics 16, 357 (1977).

1. On p. 358 , line 5 from the bottom, the expression "...e.g., $k_{1}{ }^{4}=$ $k^{2}{ }_{3}=k^{1}{ }_{3}=k_{23}$." should read "...e.g., $k_{1}{ }^{4}=k^{2}{ }_{3}=\overline{k_{3}^{1}}=\overline{k_{23}}$ "” (two last symbols under bar as complex conjugate).

2. On p. 361 the beginning of equation (2.8a) is

$$
R_{12}=-2 Z \bar{Z} \alpha_{2} \partial_{\gamma} \partial_{\bar{\gamma}} \ln \alpha+\cdots
$$

It should read

$$
R_{12}=-2 Z \bar{Z} \alpha^{2} \partial_{\gamma} \partial_{\bar{\gamma}} \ln \alpha+\cdots
$$

(alpha square).

3. On p. 362 in the first line under equation (2.9d), "...vector $e^{3 r \rightarrow 4} \ldots$ " should read “...vector $e^{3 \mu} . . . "$

4. The last two words of footnote 3 on p. 362 read "... solution only." they should read "... solutions only."

5. On p. 368 , line 9 , the expression

$$
\rho^{\prime}=\rho-2 m \ln \left(D Y^{\prime}-\bar{B}\right)-\cdots
$$

should read

$$
\rho^{\prime}=\rho-2 m \ln \left(D Y^{\prime}-B\right)-\cdots
$$

6. In some places the definition equality " $=$ def is wrongly printed as " $="$ dif 